\title{
Introduction to PNET special issue on "Resilience in future 5G photonic networks"
}

\author{
Francesco Musumeci ${ }^{1} \cdot$ Konstantinos Kanonakis $^{2} \cdot$ Paolo Monti $^{3} \cdot$ Jiawei Zhang $^{4}$
}

Published online: 27 March 2019

○) Springer Science+Business Media, LLC, part of Springer Nature 2019

Resilience will be a key aspect to address in future-generation $5 \mathrm{G}$ photonic networking, mainly due to the stringent quality of service (QoS) requirements of $5 \mathrm{G}$ services, e.g., latency, availability, energy efficiency, transport capacity, IT resources, etc. To cope with this issue, an optimized resource planning and management of photonic networks and systems is necessary.

The scope of the special issue has been well received by the research community. A total of 13 contributed papers have been submitted to the special issue, out of which 6 highquality papers have been accepted after being peer-reviewed. In addition, 6 invited papers have been also included in the special issue. The invited papers went through the same thorough peer-review process of the contributed ones. As a result, the special issue consists of a total of 12 papers. Their content is briefly summarized in the following.

In paper "Exploiting Efficiency of Ultra Dense Wavelength Switched Network for Carrying Metro Network Traffic (Invited)," the authors focus on the Ultra Dense Wavelength Switched Network (UD-WSN) paradigm as a promising candidate for resilient $5 \mathrm{G}$ metro network technology, investigating on its cost-performance trade-off.

The authors of paper "An Optical Packet Metro Architecture for Fixed Mobile Convergence in the Cloud Era (Invited)" propose optical packet switching (OPS) as a promising candidate to support future services in Metro Area Networks. An SDN-compatible flow-based transfer mode is applied in this context, and the authors study its

Francesco Musumeci

francesco.musumeci@polimi.it

Politecnico di Milano, Milan, Italy

2 Huawei Technologies, Philadelphia, USA

3 KTH Royal Institute of Technology, Stockholm, Sweden

4 Beijing University of Posts and Telecommunications, Beijing, China effectiveness for supporting local and global repair in case of failures.

In paper "Resilience-Throughput-Power Trade-off in Future 5G Photonic Networks (Invited)," the authors exploit the concepts of interleaved networks and cooperation of multiple operators to provide resource redundancy, so as to increase network resilience while mitigating deployment costs.

Paper "A Programmable Optical Network Testbed in Support of C-RAN: A Reliability Study (Invited)" provides an experimental study, where a programmable optical SDNbased testbed, supporting backhaul/fronthaul transport, is upgraded with a $1+1$ protection mechanism at the Ethernet layer.

The authors of paper "A Comprehensive Optical Mobile Fronthaul Network Towards High-Fidelity, Flexible and Low-Latency Transport (Invited)" concentrate on a variety of interesting aspects in Cloud-RAN, such as modulation formats adopted in fronthaul transmission, flexible RAN splits, optical switching fabric architectures and dynamic bandwidth allocation mechanisms.

In paper "Coexistence of OFDM and FBMC for Resilient Photonic Millimeter-wave 5G Mobile Fronthaul (Invited)," the authors propose the transmission of multiple mmWave signals as a means to improve resilience in a multi-service/ multi-operator fronthaul network.

In "Integrated Resource Optimization with WDM-Based Fronthaul for Multicast-service Beam-forming in Massive MIMO enabled 5G Networks," the authors investigate on the optimal wireless/optical resources allocation in nextgeneration radio access networks (NG-RAN) and propose a flexible WDM-based fronthaul architecture to support highcapacity and resilient transport.

On a similar way, in paper "Quality-focused resource allocation for resilient 5G network," different algorithms have been developed to perform the wireless resources scheduling to end users, based on the required QoS and targeted resilience level. 
Resilient placement of baseband processing units (BBU) has been studied in paper "Centralized vs. Distributed Algorithms for Resilient 5G Access Networks" to protect against processing (i.e., BBU nodes) failures, and in paper "Resilient BBU Placement in 5G C-RAN over Optical Aggregation Networks," where protection against link failure is also addressed.

An interesting experimental activity has been described in paper "Performance Evaluation of SDN-Controlled Green Mobile Fronthaul Using a Federation of Experimental Network Testbeds." The authors evaluate the ability of an SDNbased control to reconfigure the fronthaul traffic in order to guarantee network availability, i.e., maintain connectivity between virtual network functions (VNFs) in a virtualizedRAN environment, in case sleep-mode procedures are activated for energy efficiency purposes.

Concentrating on the physical layer, in paper " $10 \mathrm{Gbps}$ CPRI signals transmission impaired by intercore crosstalk in 5G network fronthauls with multicore fibers" the authors perform a simulative study to evaluate the outage probability of fronthaul flows in multicore fibers affected by intercore cross talk.

We hope that the research community will find this special issue interesting and stimulating for new research direction within the topic of resilience in $5 \mathrm{G}$ photonic networks.

The Editorial Board

Francesco Musumeci (Lead Guest Editor), Politecnico di Milano, Italy

Konstantinos Kanonakis, Huawei Technologies, USA

Paolo Monti, KTH Royal Institute of Technology, Sweden

Jiawei Zhang, Beijing University of Posts and Telecommunications, China

Publisher's Note Springer Nature remains neutral with regard to jurisdictional claims in published maps and institutional affiliations.

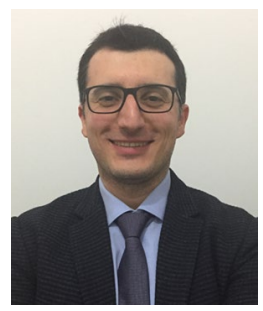

Francesco Musumeci got the M.Sc. in Telecommunication Engineering in 2009 and $\mathrm{Ph} . \mathrm{D}$. Degree in Information Engineering in 2013, both from Politecnico di Milano, Italy, where he is currently an Assistant Professor. Dr. Musumeci is author of more than 60 papers in international Journals and Conference proceedings in the field of communication networks and is co-recipient of three best paper awards. Since 2010 he has served as Technical Programme Committee member and/or reviewer for several IEEE and OSA journals and conferences in the field of optical networks. His research interests include design, optimization and performance analysis of optical networks, applications of machine learning to networking, 5G and network disaster resilience.

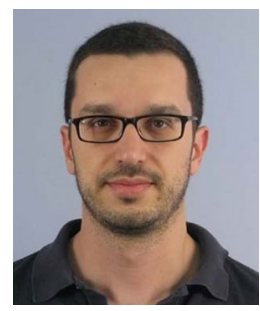

Konstantinos Kanonakis is a Senior Scientist at Huawei R\&D USA. He has extensive $\mathrm{R} \& \mathrm{D}$ experience in the fields of SDN/NFV, optical communications and data center networking. Dr. Kanonakis holds 11 U.S. Patents and has co-authored more than 60 scientific articles in international journals and conferences. He received his Ph.D. and Dipl.-Ing. Degrees in Electrical and Computer Engineering from the National Technical University of Athens, Greece.

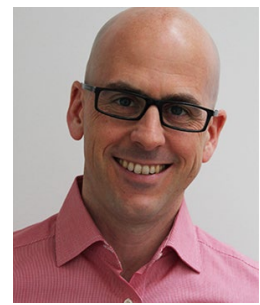

Paolo Monti is an Associate Professor at KTH Royal Institute of Technology. He holds a Ph.D. degree from the University of Texas at Dallas (USA), and a Laurea degree from Politecnico di Torino (Italy). Dr. Monti is serving on the editorial boards of the IEEE Transactions on Green Communications and Networking, of the IEEE Communications Letters, and of the IEEE Networking Letters journals. He co-authored more than 120 technical papers, with three best paper awards. His main research interests are within the architectural, technological, and sustainability challenges of $5 \mathrm{G}$ networks. Dr. Monti is a Senior Member of IEEE.

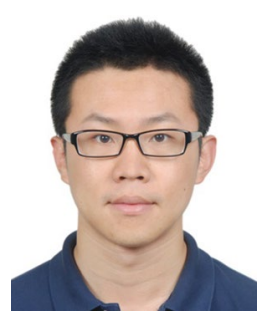

Jiawei Zhang is an Associate Professor at Beijing University of Posts and Telecommunications (BUPT). He received his Ph.D. degree from the State Key Lab of Information Photonics and Optical Communications in China. Dr. Zhang served on the TPC for IEEE DRCN 2019, and for the workshop on cloud computing systems, networks, and applications at the IEEE INFOCOM 2018, ICC 2015, and GLOBECOM 2014-2016 conferences. He first-authored more than 20 papers from journals of $\mathrm{JLT} / \mathrm{JOCN} / \mathrm{CM} / \mathrm{OE}$, and conferences of OFC/ECOC. Now, his main research interests are within the edge optical network technologies for NG-RAN and MEC. 